\title{
¿ReVOluCión O REFORMa? \\ ANUNCIOS, MEDIDAS Y COMPROMISOS A LA ESPERA DE LA REFORMA EDUCACIONAL ${ }^{1}$
}

\author{
Heidi Berner (hberner@fen.uchile.cl) \\ Facultad de Economía y Negocios
}

Cristián Bellei (cbellei@uchile.cl)

Centro de Investigación Avanzada en Educación

Universidad de Chile

\begin{abstract}
Este artículo discute el cumplimiento del programa de gobierno y otros compromisos presidenciales en relación a las políticas en materia educacional. La línea argumental se centra en que el primer año del gobierno de Sebastián Piñera se caracterizó por problemas de sistematicidad y la ausencia de un relato ordenador. Se ha hablado de paquetes de medidas, de una nueva reforma educacional e incluso de una revolución en educación. Sin embargo, las políticas recientes contienen importantes omisiones y no constituyen una estrategia coherente ni balanceada de reforma educacional. Hacia adelante se prevé que el Gobierno enfrentará una sobrecarga de su agenda en educación debido a las deficiencias de ésta y a la forma en que se ha negociado hasta ahora con la oposición.
\end{abstract}

Palabras Clave: educación, reforma, políticas públicas, Chile.

\section{REVOLUTION OR REFORM? \\ ANNOUNCEMENTS, POLICIES AND COMMITMENTS AWAITING EDUCATIONAL REFORM}

This paper discusses the fulfillment of the government's programme and other presidential commitments in the education sector. The central argument is that the first year of the Piñera government was marked by systemic problems and a lack of political narrative capable of motivating prioritization. The government has spoken of policy packages, new educational reforms, and even a revolution in education. Nevertheless recent policies contain major omissions, and constitute neither a coherent nor balanced educational reform strategy. Going forward, it seems the government will face an overloaded agenda in education due to its own internal deficiencies and the way in which negotiations with the opposition have been carried out thus far.

Keywords: education, reform, public policy, Chile.

1 Este artículo fue escrito semanas antes del inicio del movimiento estudiantil de 2011. 
Este artículo analiza los compromisos establecidos por el Presidente Piñera en materia de educación durante su primer año de Gobierno, incorporando tanto aquellos comprometidos en su discurso del 21 de mayo de 2010 (ver anexo para detalles), como en los anuncios de "nuevas medidas" y en los Protocolos de Acuerdo firmados por el Gobierno con el Parlamento con motivo de la aprobación del Proyecto de Ley de Presupuestos 2011, el Proyecto de Ley sobre Calidad y Equidad de la Educación y del Proyecto de Ley sobre el Sistema Nacional de Aseguramiento de la Calidad de la Educación Parvularia, Básica y Media y su fiscalización².

El conjunto de estas iniciativas se presentan y analizan considerando su ámbito de aplicación en el sistema educacional. En efecto, parte de las características que ha tenido la agenda gubernamental en este período ha sido una cierta falta de sistematicidad y carencia de un relato ordenador (se ha hablado de paquetes de medidas, de una nueva reforma educacional e incluso de una revolución en educación), por lo que un primer propósito de este estudio es precisamente ordenar y ponderar las iniciativas. Las preguntas que guiaron nuestro análisis fueron: más allá de los anuncios, ¿cuáles han sido los avances reales en materia de política educacional?; ¿en qué medida estos avances han dado cumplimiento al programa de gobierno y los compromisos gubernamentales?; ¿son las medidas y políticas iniciadas por el actual gobierno respuestas adecuadas a los problemas y desafios de la educación chilena?

\section{El contexto de las políticas educacionales}

El retorno a la democracia en 1990 marcó también un hito en el eje orientador de las políticas educacionales en Chile, pasando desde el foco en la expansión de la cobertura al foco en la calidad y equidad de la educación. Este cambio de prioridades tuvo su punto más alto en la Reforma Educacional iniciada en 1996, marcada por la Jornada Escolar Completa, la renovación curricular, el Sistema Nacional de Evaluación Docente (SNED) y programas de apoyo a las escuelas como el P-900 (Cox, 2003).

Aunque la implementación de esta reforma se encontraba a medio camino en 2000, el conocimiento de los insatisfactorios resultados del Sistema Nacional de Medición

2 Ésta es una de las medidas comprometidas en un "Protocolo de Acuerdo sobre Calidad y Equidad de la Educación”, firmado el 18 de enero 2011 por el Gobierno y los presidentes de los Partidos de la Concertación, con el objetivo de destrabar la tramitación del Proyecto de Ley de Calidad y Equidad de la Educación, que se transformó en la Ley 20.501 de febrero de 2011. Varias de las iniciativas analizadas en el documento fueron afectadas en diferentes grados por este Protocolo. 
de la Calidad de la Educación (SIMCE) de 1999 y de la prueba internacional Third International Mathematics and Science Study (TIMSS) de 1999, inauguró un período de enorme cuestionamiento de la reforma por parte de los líderes de opinión, los medios de comunicación y la dirigencia política. Esta crítica se agudizó luego cuando los resultados de la prueba Programme for International Student Assessment (PISA) del 2000, mostraron que prácticamente la mitad de los estudiantes chilenos de 15 años se encontraba por debajo o en el nivel 1 de dominio lector, lo que equivalía a que los alumnos poseían serias dificultades para utilizar la lectura como un instrumento para avanzar y ampliar sus conocimientos y destrezas en otras áreas; mientras que sólo un $1 \%$ se ubicaba en el nivel 5 , nivel en que los alumnos podrían manejar información presentada en textos con los que no están familiarizados, comprender textos complejos, evaluar críticamente y establecer hipótesis (UNESCO, 2000).

Durante la siguiente década, al programa inicial de la reforma se agregaría una diversidad de "medidas", las que -sin embargo- no fueron capaces de aminorar la crítica pública a la reforma -en parte por los resultados de la prueba SIMCE, que continuaban estancados en los puntajes obtenidos en $1999^{3}-$, al punto de desdibujarla casi por completo hacia mediados de la década de los 2000, todo lo cual fue asociado a una importante pérdida de relevancia de las políticas educacionales en la agenda gubernamental (Bellei, 2010).

El proceso reseñado corrió en paralelo a una creciente pérdida de importancia de la educación pública, expresada en una disminución sistemática de la matrícula de los establecimientos municipales, con el consecuente aumento de la matrícula en el sector particular subvencionado a nivel escolar, y un aumento explosivo de la matrícula de las instituciones privadas de educación post-secundaria. En efecto, en 1990 el 61\% de los alumnos de educación básica asistían a un establecimiento municipal y el 31\%, a uno particular subvencionado; en 2009 estas cifras habían cambiado, con un $45 \%$ de los alumnos de educación básica asistiendo a establecimientos municipales y un $48 \%$, a particulares subvencionados (gráfico 1).

El puntaje promedio nacional de la prueba SIMCE tanto de lenguaje y comunicación como de matemáticas fue de 250 puntos en 1990, mientras que en 2006 alcanzó a 253 y 248, respectivamente. 


\section{Gráfico 1}

Evolución de la matrícula de educación básica, por dependencia (1990-2009)

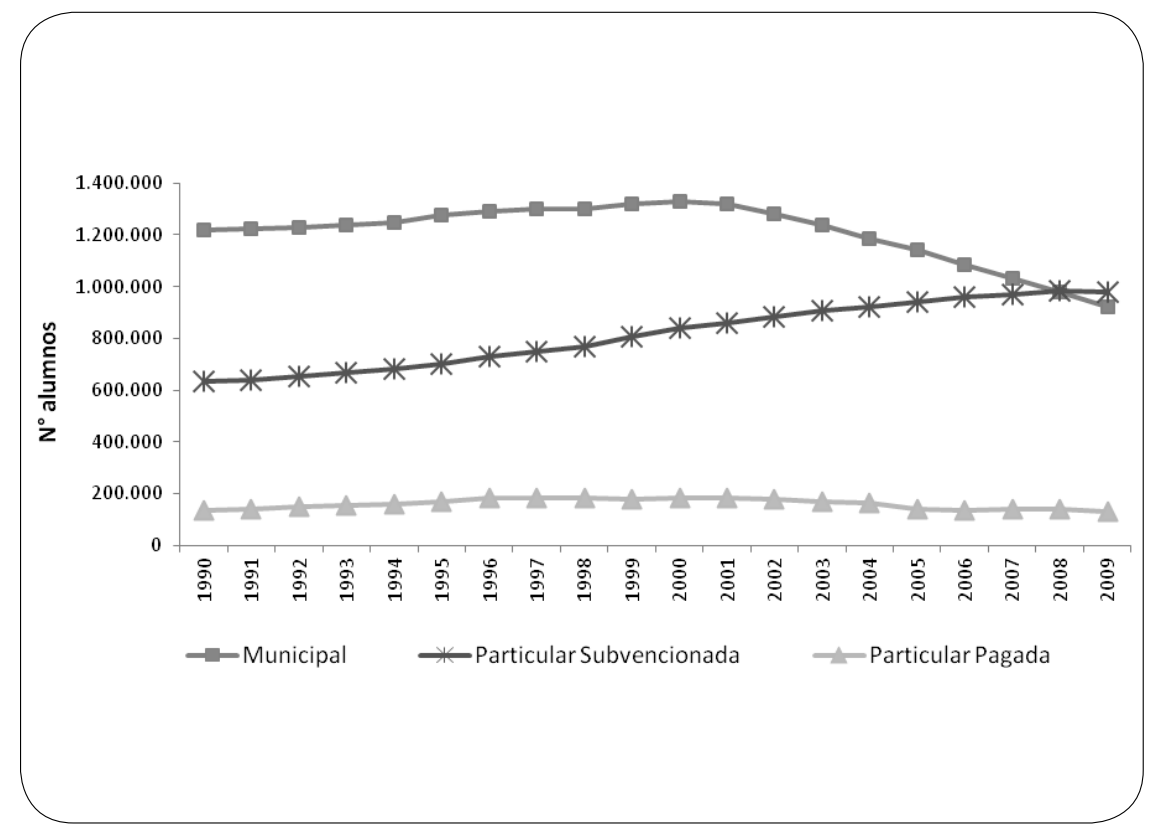

Fuente: Ministerio de Educación, 2010

En el caso de la educación media, el escenario no era muy distinto. En 1990 el 51\% de los alumnos asistía a establecimientos municipales, un 33\% a particulares subvencionados y un $8 \%$ a liceos de corporaciones; en 2009, estos porcentajes habían cambiado a $41 \%, 47 \%$ y $5 \%$, respectivamente (gráfico 2). Así, cada vez más, la sensación de "crisis" de la educación chilena se tradujo como una crisis de la educación pública.

En este contexto un movimiento social entró en escena y cambió radicalmente el escenario de la discusión pública y las políticas en el campo de la educación: la "revolución pingüina" de los estudiantes secundarios en 2006. Este movimiento tuvo al menos tres consecuencias importantes: radicalizó y generalizó la crítica y la disconformidad no sólo con las políticas educacionales, sino con el estado del sistema escolar; reposicionó la educación como prioridad en la discusión pública y en la agenda gubernamental; y abrió un espacio para la modificación institucional del sector educacional en dimensiones clausuradas por la dinámica de la elite política ${ }^{4}$.

$4 \quad$ Para un análisis de las discusiones y decisiones de política educacional gatilladas por este movimiento estudiantil, ver los artículos contenidos en Bellei, Contreras y Valenzuela (editores) (2010 y 2008) y en Brunner y Peña (coordinadores) (2007). 


\section{Gráfico 2}

Evolución de la matrícula de Educación Media, por dependencia (1990-2009)

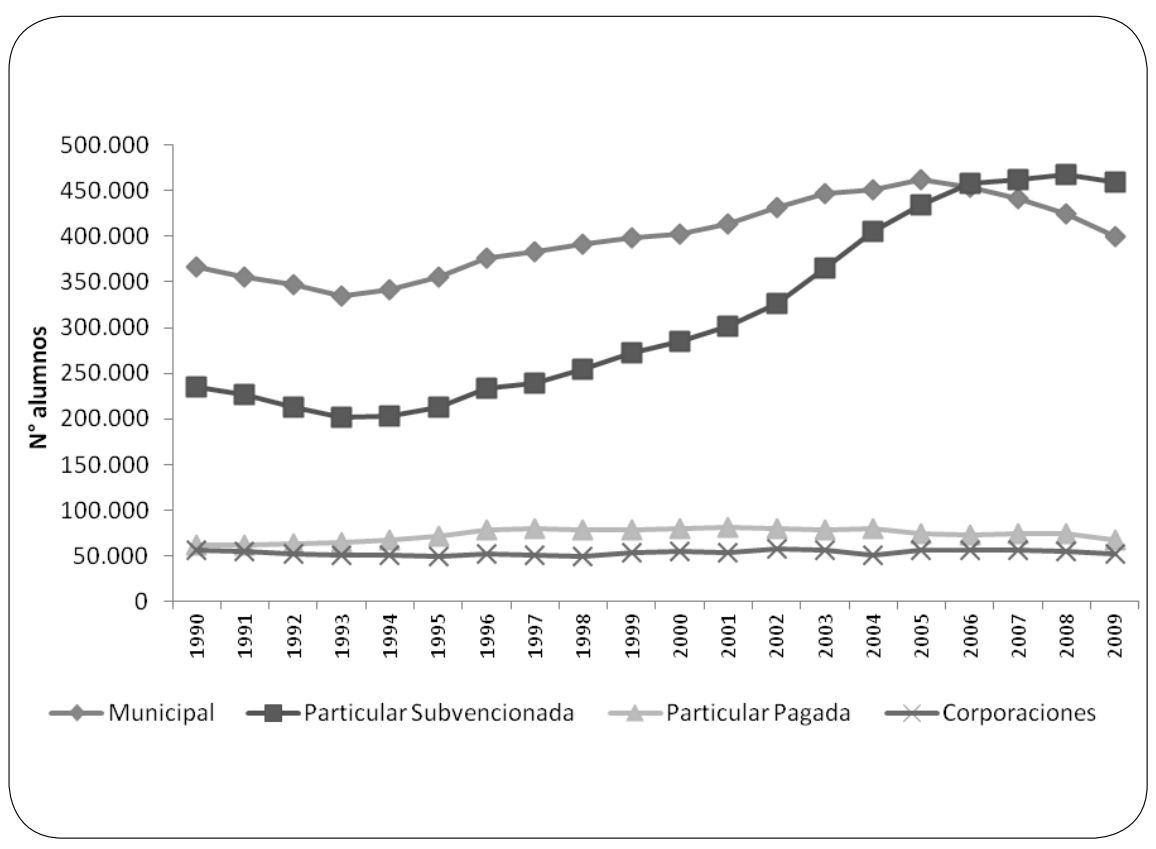

Fuente: Ministerio de Educación, 2010

Así, durante el gobierno de la Presidenta Bachelet se convocó a un Consejo Asesor Presidencial ${ }^{5}$ y se formularon tres proyectos de Ley que tenían como objetivo responder a las demandas surgidas desde la "revolución pingüina" para mejorar la calidad y equidad del sistema educativo chileno. De estos, el único que fue aprobado durante el gobierno de la Presidenta Bachelet fue la Ley General de Educación (Ley No 20.370 de septiembre de 2009), para lo cual fue necesario producir un gran “Acuerdo por la Calidad de la Educación” en 2007 entre el Gobierno, la Concertación y la Alianza (Larroulet y Montt, 2010). Recientemente ha sido aprobado, luego de pasar a tercer trámite Constitucional, el Proyecto de Ley sobre el Sistema Nacional de Aseguramiento de la Calidad de la Educación Parvularia, Básica y Media y su fiscalización (Boletín N 5.083-04).

5 Este Consejo - presidido por Juan Eduardo García-Huidobro e integrado por representantes de los estudiantes, sostenedores educacionales, docentes, académicos y otros actores sociales- sesionó durante el segundo semestre de 2006 y entregó su informe final en diciembre de ese mismo año, en el cual se plantearon un gran número de recomendaciones sobre el marco regulatorio, la institucionalidad y la calidad de los procesos internos del sistema educacional chileno, especialmente a nivel escolar. 
El tercer Proyecto de Ley de Fortalecimiento de la Educación Pública se encuentra sin urgencia, actualmente en el primer trámite constitucional en el Senado y probablemente será modificado por el gobierno del Presidente Piñera, que se comprometió al envío antes del 30 de septiembre de 2011 de un Proyecto de Ley que aborde la institucionalidad y financiamiento de la educación municipal y establezca fórmulas alternativas a la administración municipal de las escuelas y liceos.

Como se aprecia, al momento de iniciarse el gobierno del Presidente Piñera el escenario del sector educación a nivel político era de enorme dinamismo, con significativas reformas institucionales aprobadas o por aprobarse, importantes iniciativas legislativas en proceso y un intenso debate entre los principales actores nacionales. En ese denso contexto es que el gobierno planteó su programa para el sector educación.

\section{Nueva Institucionalidad para el Sistema Escolar}

Una de las principales recomendaciones del Consejo Asesor Presidencial de 2006 a la Presidenta Bachelet fue la reestructuración de la institucionalidad pública que gobierna el sector, en términos de dotarla de responsabilidades y capacidades para promover y en cierto modo "exigir" una mayor calidad a los proveedores de educación. La Presidenta envió el Proyecto de Ley sobre el Sistema Nacional de Aseguramiento de la Calidad de la Educación Parvularia, Básica y Media y la Superintendencia de Educación con este propósito, el cual luego de ser aprobado por el Senado, fue rechazado por la Cámara de Diputados en mayo de 2010, debiéndose crear una Comisión Mixta para su estudio (García-Huidobro y Bellei, 2010). La reciente aprobación por parte de la Comisión Mixta de este Proyecto de Ley, permitirá la implementación de esta nueva institucionalidad para el sistema escolar, compuesta además del Ministerio de Educación, por las dos instituciones creadas: la Agencia de Calidad de la Educación y la Superintendencia de Educación. Se trata, como en su momento se la denominó, de una nueva arquitectura del sistema escolar chileno, algunos de cuyos componentes han sido objeto de fuertes discusiones por los significativos impactos que pueda tener sobre el funcionamiento de las escuelas y liceos (Espínola y Claro, 2010; Casassus, 2010;Vanni y Bravo, 2010).

El nuevo Sistema de Aseguramiento de la Calidad establece una separación de funciones entre estas tres instituciones. El Ministerio de Educación tendrá a cargo la elaboración de la política y apoyo técnico-pedagógico a los establecimientos educaciones, la Agencia de Calidad de la Educación deberá evaluar la calidad del sistema escolar, en base a estándares y otros indicadores de calidad educativa. Por su parte, la Superintendencia deberá fiscalizar que los sostenedores de establecimientos educacionales 
reconocidos por el Estado se ajusten a la normativa educacional (leyes, reglamentos e instrucciones que dicte la Superintendencia), incluyendo la legalidad del uso de los recursos por parte de los sostenedores de establecimientos subvencionados y que reciban aporte estatal.

Para que este proyecto fuese aprobado en la Comisión Mixta, debieron ocurrir un conjunto de cambios, entre los que destaca la ampliación del concepto de calidad de la educación, que no se restringirá solo al cumplimento de estándares educativos medidos a través de pruebas estandarizadas, sino que incorpora una visión más integral y acorde con el concepto de educación definido en la Ley General de Educación, estableciendo otros indicadores de calidad educativa ${ }^{6}$. Adicionalmente, se reforzó el rol del Ministerio de Educación como órgano rector del Sistema de Aseguramiento y el encargado de prestar apoyo técnico pedagógico a los establecimientos educacionales. En este sentido, se estableció la obligación del Ministerio de elaborar un Plan de Aseguramiento de la Calidad de la Educación cada 4 años, que incorpore las acciones a desarrollar, los objetivos y metas a alcanzar, propendiendo al mejoramiento continuo del sistema educativo. También se fortaleció las competencias de la Superintendencia de Educación, estableciendo que fiscalizará el uso de todos los recursos, sean estos públicos o privados, además del cumplimiento de las instrucciones emanadas de la propia Superintendencia, y entregará capacitación a los sostenedores para que puedan realizar una adecuada rendición de cuentas. Por último destaca el alineamiento del proyecto del Sistema Nacional de Aseguramiento con las leyes de Subvenciones y Subvención Especial Preferencial (SEP), permitiendo que las categorías de clasificación de la Ley SEP (establecimiento autónomo, emergente y en recuperación) se empalmen con las categorías de ordenación creadas por el Sistema Nacional de Aseguramiento de la Calidad (desempeño alto, medio, medio-bajo e insuficiente).

Uno de los aspectos más novedosos, complejos y delicados de esta nueva institucionalidad es el sistema de sanciones a los establecimientos educacionales asociado a indicadores de logro. La nueva Ley permite el cierre de los establecimientos educacionales que -luego de cuatro años- permanezcan en un nivel de "desempeño insuficiente", en el aspecto referido al cumplimiento de los estándares de aprendizaje de los alumnos. Esto tiene varios problemas, de los cuales mencionaremos sólo dos. Como es ampliamente sabido (Koretz, 2008; Hanushek y Raymond, 2002; Ravela

\footnotetext{
6 Sin embargo, la misma ley señala que para la aplicación de sanciones a los establecimientos de bajos logros sólo se tomará en consideración los resultados en dichas pruebas, con lo cual -incoherentemente- vuelve a reducirse el concepto de "calidad educativa" en uso.
} 
et al. 2008), los indicadores de logro de las escuelas y liceos (como el SIMCE) no son una medida válida de su calidad (o "efectividad”), puesto que están -como ha demostrado la literatura especializada- fuertemente sesgados por la composición social y académica de su alumnado; en un país como Chile, que presenta niveles de segregación extremos a nivel internacional, este sesgo es aun más fuerte. Dicho de otra forma: si no se conocen las causas de los bajos puntajes SIMCE, no tiene sentido aplicar sanciones asociadas a ellos. Otro de los problemas de este sistema es que permite el cierre de establecimientos públicos aun en zonas en donde no haya más oferta pública, provocando eventualmente una completa privatización de la oferta educacional; si bien la ley obliga a resguardar el derecho a educación primaria gratuita para los alumnos que queden sin establecimientos por esta causa (i.e. las escuelas particulares subvencionadas no podrían cobrarles financiamiento compartido a ellos), no dice nada sobre los alumnos de la próxima generación ni sobre la enseñanza media. La ley debió resguardar al menos que exista oferta de educación pública a lo largo del territorio nacional, obligando a formas alternativas de reestructuración de establecimientos públicos de bajo desempeño (como de hecho la propia ley crea).

A pesar de algunos aspectos deficitarios -como los recién mencionados- el proyecto finalmente aprobado es significativamente mejor como consecuencia del trabajo de la Comisión Mixta. Ciertamente, la implementación exitosa de esta nueva institucionalidad del sistema escolar constituye un gran desafio para el Gobierno del Presidente Piñera.

\section{Políticas dirigidas hacia los docentes}

El foco más relevante de las medidas iniciadas por el actual gobierno está puesto en los profesores, lo cual ciertamente cubre un notorio déficit que tenía el Acuerdo por la Calidad de 2007 y las iniciativas impulsadas por el gobierno anterior (Bellei, Contreras y Valenzuela, 2008).

\section{Modificación al Estatuto Docente}

Respecto de la modificación al estatuto docente establecida en la Ley 20.501 (febrero de 2011), denominada Ley de la Calidad y Equidad de la Educación, cuatro aspectos se consideran importantes de destacar. El primero es la creación de un sistema de concurso público para la selección de los Jefes de Departamento de Administración de Educación Municipal y de los directores de establecimientos educacionales. Este último concurso es administrado por el Departamento de Administración de Educación Municipal (DAEM) o de la Corporación Municipal, 
según corresponda, que deberá definir el perfil del director y convocar el concurso $^{7}$. El nombramiento del director es por 5 años, estableciéndose un convenio de desempeño que debe incluir los objetivos y metas anuales, cuya evaluación de cumplimiento le corresponde al Jefe de DAEM, pudiendo solicitar la renuncia anticipada del director cuando el nivel de cumplimiento de los objetivos del convenio sea insuficiente. Respecto del concurso para seleccionar los Jefes de DAEM, este es administrado por el Consejo de ADP, siguiendo el mismo procedimiento establecido para el nombramiento de altos directivos públicos de segundo nivel jerárquico, designándose una Comisión Calificadora integrada por el sostenedor, un miembro del Consejo de ADP y un director de establecimiento educacional municipal de la respectiva comuna, elegido por sorteo de entre los directores que hayan sido designados por concurso ${ }^{8}$. Al igual que en el caso del director, el nombramiento será por 5 años, estableciéndose un convenio de desempeño con el sostenedor, quien deberá evaluar anualmente el cumplimiento de los objetivos, pudiendo pedir la renuncia anticipada por incumplimiento de los mismos.

El segundo es la entrega de atribuciones para que los directores de establecimientos municipales nombren a su equipo directivo de exclusiva confianza (Subdirector, Inspector General, y Jefe Técnico Profesional), pudiendo designar a profesionales que no pertenezcan a la dotación de docentes de esa comuna. También, podrán proponer anualmente al sostenedor despedir al 5\% de los profesores, de entre quienes resulten "mal evaluados" (aproximadamente el tercio inferior) en la evaluación docente del Estatuto. Los docentes despedidos por este motivo tendrán derecho a una indemnización fijada en UTM que varía dependiendo del valor del promedio de las últimas 12 remuneraciones, con un máximo de 143,2 UTM9.

7 El proceso técnico de evaluación de los candidatos podrá apoyarse en asesorías externas registradas en la Dirección Nacional de Servicio Civil, con el fin de preseleccionar los candidatos a entrevistar por la Comisión Calificadora creada para estos efectos e integrada por el Jefe de DAEM, un miembro del Consejo de Alta Dirección Pública (ADP) y un docente de otro establecimiento educacional perteneciente a la dotación del municipio, elegido por sorteo entre aquellos docentes de la red de Maestros de Maestros o acreditados como profesores de excelencia pedagógica. Esta Comisión deberá presentar los postulantes seleccionados al sostenedor (mínimo tres, máximo cinco), quien toma la decisión.

8 El proyecto enviado por el gobierno establecía que debía ser un funcionario de más alta jerarquía del mismo municipio, sin embargo fue modificado por una indicación de los Diputados.

9 El proyecto enviado por el gobierno establecía que la indemnización correspondía al 50\% de la remuneración del último mes y que los docentes podían ser despedidos en base a una evaluación diseñada por el sostenedor, sin embargo ambos aspectos fueron modificados por indicaciones de los Diputados. 
Tercero, se establecen aumentos de remuneraciones para el Jefe de DAEM y los directores, a través de una asignación especial que varía dependiendo de la matrícula municipal y su nivel de vulnerabilidad ${ }^{10}$. Adicionalmente, se aumenta el monto de la Asignación de Excelencia Pedagógica de los docentes de aula del sector subvencionado, aunque se disminuye su duración de 10 a 4 años. El monto de la asignación irá de $\$ 50.000$ a $\$ 150.000$ mensuales para los docentes mejor evaluados que tengan una jornada de 44 hrs. semanales ${ }^{11}$.

Cuarto, se establece una bonificación de retiro para profesores del sector municipal, que renuncien a su contrato y que al 31 de diciembre de 2013 tengan 60 o más años si son mujeres y 65 o más años si son hombres ${ }^{12}$. También se entrega un "bono especial para docentes jubilados", que hayan trabajado por un mínimo de 10 años en establecimientos municipales. El valor máximo del bono sería de $\$ 2$ millones para docentes con pensiones y beneficios previsionales inferiores a $\$ 150.000$ mensuales brutos. Para solventar los mayores gastos asociados a las indemnizaciones de los docentes y al plan de retiro, se crea un fondo con $\$ 20$ mil millones al que pueden postular los municipios.

\section{Examen de excelencia profesional docente y asignación de excelencia pedagógica inicial}

El gobierno ingresó en noviembre de 2010 al Senado un proyecto de Ley que crea el Examen de Excelencia Profesional Docente y la Asignación de Excelencia Pedagógica Inicial ${ }^{13}$. El objetivo del examen, conocido como Prueba Inicia, es medir los conocimientos disciplinarios de los profesores recién egresados; sus contenidos deberán ser aprobados por el Consejo Nacional de Educación y sus resultados -a nivel de institución formadora y carrera- deberán ser públicos (Manzi, 2010). El examen

10 En la Cámara de Diputados se aprobó una indicación para que la asignación no sólo aumentase con el tamaño, sino también con el nivel de pobreza de la matrícula.

11 Creada por la Ley 19.715 de enero de 2001. De acuerdo al Informe Financiero del proyecto Ley de Calidad y Equidad de la Educación, esta asignación beneficiaría a 4.500 docentes en 2011, 6.500 en 2012 y 8.500 en 2013.

12 Con un máximo de $\$ 20$ millones para profesores con 11 años o más de servicio y contrato de 44 hrs., y que presenten su intención de retiro antes de 1 de agosto de 2011. Quienes presenten su intención de retiro entre el 1 de agosto de 2011 y el 1 de diciembre de 2012 recibirán sólo el $80 \%$ del monto de bonificación que les corresponda. Luego del plazo, se faculta al sostenedor para declarar vacantes la totalidad de horas de contrato de los docentes que, cumpliendo con los requisitos del plan de retiro, no lo soliciten.

13 Boletín $\mathrm{N}^{\circ}$ 7327-04; el proyecto se encuentra sin urgencia, en su primer trámite constitucional en el Senado. 
será voluntario, aunque haberlo rendido será un requisito para ejercer en los establecimientos de educación subvencionados por el Estado. Asociado a este examen se crea una asignación de excelencia pedagógica inicial que aumenta las remuneraciones de los nuevos docentes que ejerzan en establecimientos subvencionados por un mínimo de 22 hrs. semanales, y que obtengan un puntaje dentro del 30\% superior: el 10\% de más altos puntajes recibirá $\$ 150.000$, el siguiente $10 \%, \$ 100.000$, y el último $10 \%$, $\$ 50.000$, por tres años, para docentes con contrato de $44 \mathrm{hrs}$. semanales. De acuerdo al informe financiero de este proyecto de Ley serían 16.426 los futuros profesionales que rendirían el examen en 2011.

\section{Beca Vocación de Profesor}

Aunque en estricto sentido se trata de una modificación y expansión de un programa previamente existente ${ }^{14}$, también ha sido ampliamente difundida la beca que financia el 100\% del arancel 2010 a jóvenes que obtengan 600 o más puntos en la PSU y que postulen como primera opción a estudiar pedagogía. Los jóvenes deberán matricularse en carreras acreditadas o en proceso de acreditación y que posean puntajes de corte de 500 o más puntos PSU. Los jóvenes deberán comprometerse a obtener el título profesional y trabajar por tres años en un establecimiento subvencionado (municipal o particular). Adicionalmente, de entre los mismos jóvenes, quienes obtengan 700 o más puntos en la PSU y posean un promedio de notas en la enseñanza media igual o mayor a 6.0, se les otorgará una beca de $\$ 80.000$ mensuales de libre disposición; y a quienes obtengan más de 720 puntos en la PSU, tendrán asegurado un semestre de intercambio en el extranjero.

Miradas en su conjunto, las medidas referidas a la profesión docente tienen dos ejes básicos: la flexibilización de la gestión de los recursos humanos en el sector municipal y el uso de incentivos monetarios asociados a la aplicación de pruebas estandarizadas. Respecto de la primera dimensión, si bien ésta apunta a resolver algunos problemas de eficiencia en la gestión del sector municipal (agudizados por la acelerada pérdida de matrícula), al no abordarse las causas de este problema ni modificarse la estructura institucional de ese subsector, difícilmente se puede prever

14 Las becas para estudiantes de pedagogía existen desde fines de los noventa. Financiaban la totalidad o parte del arancel anual de la carrera con un monto máximo de $\$ 1.150 .000$, pudiendo postular jóvenes con un puntaje PSU promedio igual o superior a 600 puntos, con un promedio de notas de Enseñanza Media igual o superior a 6,0 y que postulen como primera opción a una carrera acreditada de pedagogía en las Universidades del Consejo de Rectores. 
su mejoramiento ${ }^{15}$. Respecto de la segunda, no existe evidencia científica suficiente para afirmar que los test estandarizados identifican a los docentes más efectivos ni que los incentivos monetarios producen un aumento de la efectividad docente (Umansky, 2005; Hunt, 2009). Finalmente, a pesar del enorme consenso existente sobre la necesidad de reformas mayores en este campo, es evidente que el gobierno ha priorizado este tipo de medidas "indirectas", postergando otras como la creación de una carrera profesional docente, el mejoramiento y regulación de la formación inicial docente, la formación continua y el mejoramiento de las condiciones laborales de los profesores (sobre este punto recomendamos: Cox, Meckes y Bascopé, 2010; Bellei y Valenzuela, 2010; Consejo Asesor de 2006, Panel de Expertos 2010 y variados informes internacionales, como OECD 2009, y Barber y Mourshed 2007).

En esta misma línea, por iniciativa de la oposición, el gobierno se comprometió -en el referido "Protocolo de Acuerdo" de enero 2011- a enviar antes del $1^{\circ}$ de marzo de 2012 un Proyecto de Ley que permita modernizar "la carrera profesional docente buscando que ella se adecue a la realidad actual y considere las necesidades de formación continua de los docentes".

\section{Diversas iniciativas para mejorar la calidad y equidad de la educación}

Durante el primer año, el Gobierno anunció una variedad de "nuevas medidas" dirigidas a diferentes aspectos del mejoramiento educacional. Tres son, a nuestro juicio, las principales.

Primero, la creación de 60 Liceos de Excelencia ${ }^{16}$ a lo largo del país, en un intento por expandir el modelo académicamente selectivo del Instituto Nacional. Aunque en un principio se dijo que éstos serían liceos públicos, el gobierno terminó financiando también algunos liceos privados. Aunque esta medida ha sido criticada por algunos académicos y actores sociales por tener el potencial de aumentar la segregación de la población escolar, la fuerza simbólica de “expandir" el Instituto Nacional ha sido muy fuerte. Con todo, acogiendo en parte las críticas, el gobierno incluyó algunos mecanismos en su funcionamiento destinados a mitigar el impacto segregador de esta medida (por ejemplo, que el 30\% de su matrícula sea seleccionada por sorteo).

15 En efecto, es una falta de rigor atribuir al Estatuto Docente la supuesta inferior calidad de la educación municipal: en promedio y controlando por las diferencias de alumnado, el sector particular no produce resultados superiores (Bellei, 2009).

16 En el discurso del 21 de mayo se había anunciado 50 Liceos de Excelencia, de los cuales 25 deberían estar funcionando en 2011; el 22 de noviembre el Presidente señaló que serían 60, 30 de ellos en 2011. 
Segundo, la intensificación de la difusión de los resultados SIMCE, especialmente hacia los padres y apoderados. Para ello el gobierno implementó un sofisticado sistema de mapas comunales (en versión internet, pero enviado también por carta a los hogares), que identifica a los establecimientos con los colores de un semáforo, según si se encuentran por sobre, bajo o en el promedio SIMCE nacional. Esto con el propósito de hacer más eficiente el mercado escolar. La medida fue enormemente criticada por académicos y actores del campo educacional, por cuanto la información entregada, al estar sesgada por las características del alumnado, no permite conocer la efectividad real del establecimiento (por lo que orienta a la demanda con información inválida) y tiene el potencial de aumentar la ya existente segregación escolar. A pesar de haber inicialmente defendido este instrumento, el Gobierno finalmente accedió a perfeccionar el sistema en lo referido al sesgo socioeconómico que éste introduce.

Tercero, un cambio en los Planes y Programas de Estudio -con aprobación del Consejo Nacional de Educación- que además de ciertas modificaciones de contenido, aumenta las horas semanales de ciertas asignaturas (Lenguaje de 5 a 7 entre $5^{\circ}$ básico y $2^{\circ}$ medio; Matemáticas de 5 a 6 entre $5^{\circ}$ y $8^{\circ}$ básico, y de 5 a 7 en $1^{\circ}$ y $2^{\circ}$ medio; e Inglés de 2 a 3 en $5^{\circ}$ y $6^{\circ}$ básico), disminuyendo horas dedicadas a Historia y Ciencias Sociales, y Tecnología. Esta medida ha sido ampliamente criticada en lo referido a la disminución de las horas de estudio de ciertas asignaturas consideradas de gran relevancia, más aun cuando el horario extendido de la jornada escolar completa hace innecesaria dicha disminución. También en este aspecto el Gobierno accedió finalmente a revertir parcialmente la medida, en el marco de las negociaciones del Protocolo de enero de 2011.

Como se ve, se trata de iniciativas de mérito variable (mayoritariamente cuestionadas por la comunidad experta), focalizadas en aspectos muy específicos del funcionamiento del sistema educacional y que no están articuladas entre sí. En otras palabras, como de hecho se las ha comenzado a nombrar, son "medidas" en cierto modo asistemáticas y en ningún caso parte de lo que se podría denominar una nueva "reforma educacional" impulsada por el Gobierno.

\section{Financiamiento de la Educación}

Uno de los compromisos presidenciales más relevantes en educación es duplicar la subvención escolar en un plazo de 8 años. En esta dimensión, el gobierno ha impulsado dos proyectos de Ley: el primero propone un aumento en un $20 \%$ promedio 
de la Subvención Escolar Preferencial (SEP) ${ }^{17}$; el segundo -la Ley de la Calidad y Equidad de la Educación- incrementa en 1,5\% promedio el valor de la subvención escolar a partir del 1 de enero de $2012^{18}$. Nótese que aunque en rigor este proyecto afectaría sólo al sector municipal, el aumento de la subvención general beneficiará también al sector privado subvencionado.

Adicionalmente, destacan dentro de los cambios incorporados al Proyecto de Ley de Calidad y Equidad producto del Protocolo de Acuerdo, el aumento de la cobertura de la Subvención Escolar Preferencial (SEP) hasta cuarto medio en forma gradual, partiendo en 2014 con primero medio, alcanzando a cuarto medio en 2017; el incremento en un 20\% de la subvención de concentración de la SEP, y el aumento de hasta en un 30\% en la Asignación Variable de Desempeño Individual, para los docentes que se desempeñen en establecimientos con alto índice de vulnerabilidad.

Respecto de la subvención educacional regular, el Gobierno se comprometió, en el mencionado Protocolo de Acuerdo, a crear una subvención escolar adicional para la clase media. No existe plazo ni definición de clase media en el Protocolo.

Otro aspecto relevante es el reemplazo del Programa de Extensión de la Jornada Escolar Completa por el Programa de Infraestructura Educacional en la Ley de Presupuesto de 2011, incorporando $\$ 337.006$ millones en 2011. De estos recursos, el 59\% permite el financiamiento de construcciones, reparación y/o normalización de establecimientos subvencionados ubicadas en zonas afectadas por el terremoto. Adicionalmente, se consideran $\$ 13.687$ millones para financiar la construcción, reposición, reparación y/o ampliación, así como también la adquisición de equipamiento y mobiliario de los 30 Liceos Bicentenarios de Excelencia comprometidos para 2011.

Ciertamente la emergencia causada por el terremoto de febrero de 2010 obligó a redefinir el itinerario (y quizás la meta) del aumento de la subvención escolar, por lo que en ese contexto priorizar la reposición de la infraestructura educacional y el aumento de subvención focalizado en los más pobres parecen bien justificado. Con todo, más allá de la cuestión del monto de la subvención estatal, un tema pendiente

17 Ley 20.248 de 2008; su modificación se encuentra en segundo trámite Constitucional en la Comisión de Hacienda de la Cámara de Diputados, Boletín N 7187-04. El proyecto además flexibiliza el uso y rendición de estos recursos por parte de los sostenedores.

18 El proyecto haría efectivo este aumento a partir de 2011, sin embargo, debido a una indicación en la Comisión de Hacienda de la Cámara de Diputados, parte de estos recursos se destinarían a aumentar la subvención por concentración de pobreza de la SEP en 2011, postergando el aumento general para 2012. 
es el mejoramiento del sistema de financiamiento a la educación escolar, dado que la "subvención por alumno atendido" tal y como está diseñada presenta varias limitaciones para la equidad y la eficiencia (Consejo Asesor Presidencial, 2006). El gobierno ha anunciado su voluntad de conformar una comisión técnica para estudiar este asunto durante 2011.

\section{Iniciativas para el Fortalecimiento de la Educación Municipal}

Uno de los procesos más salientes que está experimentando el sistema escolar chileno es su acelerada privatización, producto de la pérdida de relevancia de la educación pública, a cargo de los municipios desde inicios de los 1980. Las causas de esta paulatina reducción de la educación escolar pública son múltiples y fueron analizadas detenidamente por el Consejo Asesor Presidencial de 2006, el cual recomendó impulsar una importante reestructuración de este subsector. Sin embargo, ésta no ha sido un área priorizada por las políticas educacionales. En cambio, los sucesivos gobiernos han respondido a la demanda de los alcaldes por mayores recursos con iniciativas ad hoc.

Así, como parte de las medidas comprometidas por el Gobierno del Presidente Piñera en el "Protocolo de Acuerdo sobre Calidad y Equidad de la Educación" del 18 de enero 2011, se generaron un conjunto de indicaciones al Proyecto de Ley de Calidad y Equidad de la Educación que permitieron la creación de dos fondos: el Fondo Transitorio de Apoyo a la Mejora de la Calidad de la Educación Municipal, cuyos recursos se concursarán entre municipios, los que deben presentar un plan de mejoramiento que incluya metas específicas de gestión, financiamiento y resultados educativos, siendo los recursos entregados en cuotas contra el cumplimiento de dichas metas ${ }^{19}$; y el Fondo de Apoyo a los Municipios para el Financiamiento del Plan de Retiro de 13.000 docentes que tienen o cumplen su edad de jubilar hasta diciembre de $2012^{20}$.

Los problemas de la educación pública, sin embargo, no se limitan a la falta de recursos. Adicionalmente, como parte del mismo Protocolo de Acuerdo el Gobierno se

19 El Fondo Transitorio de Apoyo a la Mejora de Calidad de la Educación Municipal cuenta con $\$ 45.000$ millones, distribuidos así: \$10 mil millones en 2011, \$15 mil millones en 2012 y \$20 mil millones en 2013.

20 El Fondo de Apoyo a los Municipios cuenta con $\$ 60.000$ millones, los que se otorgarán entre 2011 y 2014. La mitad de estos recursos se distribuirá utilizando criterios de matrícula y vulnerabilidad de los municipios, existiendo un porcentaje de recursos que se repartirá en partes iguales entre todos los municipios. La otra mitad se repartirá entre aquellos municipios que no pueden financiar su plan de retiro con adelanto de subvención. 
comprometió a enviar antes del 30 de septiembre de 2011 un Proyecto de Ley que aborde la institucionalidad y modo de financiamiento de la educación municipal, y que establezca fórmulas alternativas a la administración municipal de las escuelas y liceos. Hasta aquí, al margen de declaraciones genéricas de las autoridades, el Gobierno no ha sido explícito en comunicar su propuesta de cambio institucional para la educación pública ni menos ha aclarado cuán distinta será de aquella contenida en el Proyecto de Ley de Fortalecimiento de la Educación Pública ${ }^{21}$ enviado por el Gobierno anterior ${ }^{22}$.

\section{Educación Superior}

Aunque el gobierno ha anunciado que emprenderá ciertas iniciativas en este nivel educacional, lo cierto es que hasta aquí la preocupación por él ha sido menor (especialmente si se la compara con la atención dada a la educación escolar). Así, por ejemplo, se publicitó recientemente la creación de un Sistema de Información de la Educación Superior, que en realidad constituye un refinamiento de sistemas existentes previamente. También se ha anunciado cambios a la PSU (que se rinda dos veces al año, que el puntaje sea válido por 2 años), los cuales no dependen del gobierno. Es decir, está pendiente -si la va a haber- conocer la política hacia la educación superior.

Respecto de los recursos destinados al financiamiento de la Educación Superior, existe un aumento del 9\% (\$774.480 millones) en el presupuesto 2011, destacando el aumento de $22 \%$ de los recursos para financiar becas. El Fondo de Desarrollo Institucional destinado a instituciones del Consejo de Rectores aumentó en 20\% (\$8.575 millones en 2011), y se creó una línea de este mismo Fondo de \$2.144 millones para ser concursados entre las universidades privadas autónomas, los institutos profesionales y los centros de formación técnica acreditados (inicialmente el gobierno propuso la creación de un único fondo para todas las instituciones de educación superior). Por último, destaca la firma del Protocolo de Acuerdo para el Despacho del Proyecto de Ley de Presupuestos del Sector Público para 2011 (entre el Ministro de Hacienda y la Comisión Especial Mixta de Presupuestos, 08 de noviembre de 2010), en el cual el gobierno compromete $\$ 8.500$ millones en

21 Proyecto sin urgencia que se encuentra actualmente en el primer trámite constitucional en el Senado.

22 Además del mencionado Consejo Asesor Presidencial de 2006, existen varias publicaciones recientes con diagnósticos y propuestas sobre cómo fortalecer la educación pública chilena; ver por ejemplo, Beyer yVelasco, 2010; Bellei, González yValenzuela, 2010; García-Huidobro, 2010; Elacqua, Martínez y Aninat, 2010; y Marcel et al., 2009. 
2010 para apoyar las labores de reconstrucción de las Universidades del Consejo de Rectores. Adicionalmente, se compromete al envío durante el primer semestre de 2011 de un proyecto de Ley que permita flexibilizar y desburocratizar la gestión financiera de las Universidades Estatales, asociándola a convenios de desempeño. Finamente, señala que "Con el objeto de fomentar las ciencias sociales, el Ministerio de Educación realizará aportes a la Universidad de Chile por $\$ 5.310$ millones en diciembre de 2010, y luego el mismo monto en enero de 2012, 2013 y 2014, según la aprobación de los avances del convenio que para este efecto suscriban las partes".

Posteriormente, en el Protocolo de Acuerdo de enero de 2011, el Gobierno se comprometió a estudiar mecanismos de mejora al sistema de acreditación de instituciones de educación superior, en particular para el caso de carreras de pedagogía, y a crear un programa especial de incentivo al 5\% de los mejores egresados de enseñanza media de sectores vulnerables para acceder a la educación superior, independientemente de su puntaje en la PSU.

También en este campo (que experimenta una expansión y diversificación aceleradas), actores y especialistas han propuesto y discutido largamente reformas mayores a su institucionalidad y formas de gobierno, mecanismos de financiamiento, y sistemas de aseguramiento de la calidad y fiscalización, al menos (Consejo Asesor Presidencial para la Educación Superior, 2008). Sin embargo, las autoridades educacionales no han impulsado cambios que impliquen una reestructuración del nivel de educación post secundario. El gobierno del Presidente Piñera ha sido insistente en manifestar su voluntad por romper dicha inercia, lo cual requiere no sólo de una propuesta consistente con el negativo diagnóstico, sino de una enorme dosis de capacidad negociadora y liderazgo, dados los enormes intereses corporativos y la fuerza de los actores involucrados en este campo.

\section{Discusión y perspectivas}

En esta sección final quisiéramos poner el conjunto de la política educacional desplegada por el Gobierno del Presidente Piñera en una perspectiva de mediano plazo. Para ello, realizaremos dos tipos de análisis. El primero, sobre la envergadura de la agenda de políticas educacionales y su vínculo con los procesos políticos de negociación y acuerdo. El segundo, referido al sentido más general que las políticas educacionales, y en cierto modo la institucionalidad educacional chilena, han ido adquiriendo. 
En primer lugar: sobre el tamaño y complejidad de la tarea en educación. La pregunta estándar para un análisis de este tipo parece ser: ¿Cuánto ha cumplido el Gobierno con lo prometido en educación? Sin embargo, a nuestro juicio, ésta no es actualmente la pregunta más relevante en el campo de las políticas educacionales en Chile.

En su primer año, el gobierno -al igual que sus predecesores- ha priorizado la educación escolar por sobre la educación superior. Ahora bien, dentro de la amplia y compleja agenda de cambios en la educación escolar, el gobierno ha puesto más esfuerzos en "abrir nuevos temas", que en "amarrar" los abiertos post-revolución de los pingüinos e incluidos en el Acuerdo por la Calidad de 2007 (cuyo principal gestor fue precisamente el actual Ministro Secretario General de la Presidencia, Cristián Larroulet). Dicha Agenda incluye tanto implementar los cambios introducidos por la Ley General de Educación como diseñar y poner en marcha la nueva institucionalidad del sistema escolar, partiendo por la puesta en funcionamiento de la Superintendencia de Educación y la Agencia de la Calidad, todo lo cual constituye un enorme rediseño institucional y un gran esfuerzo de gestión para el Ministerio de Educación. En cualquier otro contexto nacional, sólo este primer componente de la Agenda "heredada" de política educacional sería suficiente para considerarse en medio de una "gran reforma" educativa.

No obstante, el Gobierno ha impulsado un gran número de iniciativas adicionales en varios aspectos de la educación escolar: evaluación de profesores, pruebas SIMCE, cambios curriculares, liceos de excelencia, entre otros. Anunciadas con una enorme (en verdad, excesiva) retórica y un fuerte énfasis en la dimensión comunicacional (postergando en cambio la negociación con actores relevantes), las variadas iniciativas aquí analizadas no constituyen en rigor una nueva "reforma educacional" ni menos una "revolución" como las denominó el Gobierno en su momento, sino un conjunto de medidas de mérito variable (varias de ellas bastante discutibles, como fue expuesto) y relativamente desconectadas unas de otras. Más aun, en el proceso de negociaciones políticas para hacer viables algunas de estas iniciativas (principalmente con la Concertación, pero también con algunos actores del campo educacional), el Gobierno ha debido asumir nuevos compromisos en temas tan decisivos como la creación de una carrera profesional docente o el rediseño del sistema de administración de los establecimientos municipales. A lo anterior se suma la prioridad anunciada por el Gobierno para la educación superior en el período que viene. Como queda claro, la suma de estos nuevos compromisos (los autoim- 
puestos y los asumidos posteriormente) configura una agenda adicional de políticas educacionales casi tan demandante como la agenda que antes llamamos "heredada". Paradojicamente, entonces, aunque el gobierno ha sido muy activo en cumplir con los anuncios realizados al inicio de su período, la tarea que le queda por delante en el sector educación es mucho mayor que la que tenía al comenzar su mandato. ¿Cómo se entiende esta paradoja?

Lo primero es comprender que en materias de política educacional (especialmente las referidas a la calidad y la equidad educativas), la realización de una determinada política no se agota con la dictación de una ley o el lanzamiento de un programa: los desafios tradicionalmente llamados "de implementación" son consustanciales a la gestión de la política misma. En esta categoría se podrían agrupar todos los componentes asociados a la Subvención Escolar Preferencial, la Ley General de Educación, la Superintendencia de Educación y la Agencia de la Calidad de la Educación. Todas estas "iniciativas" son en verdad procesos de cambio institucional, de gestión y educacionales de marca mayor, que requieren la toma de decisiones técnico-políticas con consecuencias determinantes sobre el rumbo que estos procesos tomarán y su potencial efectividad.

Una segunda fuente que alimenta esta abultada agenda de políticas son las nuevas prioridades que el actual gobierno ha intentado impulsar. El Presidente Piñera ha definido al cambio educacional como "la madre de todas las batallas" que él espera liderar, para lo cual -ya se ha dicho- no se ha escatimado en el uso de la retórica y en la búsqueda de golpes comunicacionales. Es difícil convencer a la ciudadanía de que se está impulsando una misión semejante solamente dando continuidad a la agenda heredada de los gobiernos dirigidos por la actual oposición. Aunque las transformaciones en curso hayan sido fruto de amplios acuerdos políticos, el deseo por marcar un punto de inflexión, por poner un sello propio, es muy fuerte. Con multiplicidad de medidas en diferentes dimensiones (e.g. énfasis en el uso de tests, foco en los profesores), el Gobierno parece haber ensayado diferentes alternativas para cumplir este propósito, con poco éxito hasta el momento. La promesa de priorizar la educación superior en la siguiente etapa se puede interpretar como parte de esta búsqueda, aunque nuevamente presionado por masivos movimientos ciudadanos.

Finalmente -y ésta es la cuestión más interesante a nuestro juicio-, existe un tercer afluente de este caudal de políticas en educación: las negociaciones con los actores políticos y educacionales. Durante su primer año de administración (como queda claro de la lectura de nuestro análisis), el Ministro Lavín ha estado involucrado en 
una gran variedad de negociaciones y acuerdos, en los que, para dar viabilidad a sus nuevas iniciativas, ha debido asumir compromisos adicionales, sobre aspectos tan ajenos a las tradicionales posturas de la Alianza gobernante como comprometerse a reformar y fortalecer la educación pública, o crear una carrera profesional docente. La búsqueda de acuerdos políticos amplios en educación es una condición relevante para dar sostenibilidad de largo plazo a reformas y políticas que requieren tiempos prolongados para desplegarse y ser eventualmente efectivas. Estos acuerdos nacionales fijan prioridades tras las cuales el conjunto de las fuerzas políticas se alinean; en otras palabras, debieran contribuir a reducir y focalizar la agenda gubernamental en educación. Sin embargo, no creemos que el proceso en curso en este último período se pueda describir de esa forma. Se ha tratado, más bien de un proceso de transacciones parciales en que más que consensuar la prioridad compartida, ambas partes han aceptado las prioridades del otro a condición de que las propias sean también aceptadas. Así, el proceso de negociación ha derivado en una virtual bola de nieve (e.g. el Protocolo de Acuerdo Gobierno-Oposición que se firmó para destrabar la Ley de Calidad y Equidad, contiene más de 40 medidas acordadas).

En segundo término: ¿Qué podemos decir sobre el sentido más general que ha tomado el curso de la reforma educacional chilena?Vista en perspectiva, la última media década marca un punto de inflexión en el desarrollo de nuestro sistema escolar: el modelo de libre mercado deja de ser su referente institucional. Son muy pocos los que aún siguen esperando que la sola competencia entre escuelas y la privatización vayan a producir la educación de calidad para todos que el país necesita. Ése es, a nuestro juicio, el principal valor histórico que tienen el "Acuerdo por la Calidad" y el "Protocolo de Acuerdo", suscritos por la Concertación y la Alianza en 2007 y 2011, respectivamente. Sacudidos ya del sueño de la auto-regulación, entramos a las turbulentas aguas de la política educacional.

El desafio básico del paradigma que comienza a emerger consiste en configurar un complejo arreglo institucional en que el Estado cumple múltiples roles para garantizar la calidad y la equidad educativas: define estándares de calidad y reglas del juego, evalúa y exige responsabilidades, apoya e implementa políticas, financia y fiscaliza a los proveedores, informa a la comunidad e interviene en situaciones deficitarias, todo para asegurar que escuelas y liceos provean una educación de calidad para todos. En términos sintéticos, se busca combinar lo más sabiamente la "presión" sobre las instituciones educativas para que mejoren su calidad, con el necesario "apoyo" para lograrlo; en otras palabras, disponer los “incentivos" para que los agentes educacionales cumplan con estándares cada vez más exigentes y generar en ellos las 
"capacidades" para responder productivamente a dichos incentivos. La dificultad mayor radica en encontrar el equilibrio entre estos dos polos, considerando que dicho equilibrio depende críticamente de las condiciones existentes en el sistema educacional del que se trate.

Desde esta perspectiva, ¿qué tan equilibrada es la agenda legislativa y de políticas educacionales que se ha ido priorizando en Chile? No mucho.Veamos brevemente tres ejemplos para fundamentar este juicio. El primero es la notable postergación de la educación pública: aunque el fracaso de la municipalización es evidente, los tomadores de decisión aun no se deciden a actuar agresivamente sobre ella para fortalecerla, como hacen prácticamente todos los países de la OECD. Así, la educación pública es la gran pieza ausente (postergada también por los gobiernos anteriores), a pesar de que experimenta una crisis de enorme gravedad, que según muchos pone en riesgo su propia existencia. El segundo caso es el de los profesores: es sabido que el estatus de la docencia en Chile es bajo para competir con las demás profesiones, pero no ha habido un abordaje integral para hacer atractiva la carrera docente; más aun, la desregulada explosión de la matrícula de las carreras de pedagogía en la última década se ha hecho sin respetar criterios mínimos de calidad, lo que no augura un buen futuro.

El último ejemplo es el de las escuelas privadas subvencionadas: el desarrollo de este sector no ha seguido ningún criterio estratégico para el mejoramiento del sistema, sino que ha respondido a los intereses de los sostenedores privados, derivando en situaciones de sobreoferta, aumentando la segregación de los alumnos y afectando negativamente a los establecimientos municipales; lamentablemente, la nueva legislación del sector no modifica este panorama en lo sustancial, porque confia completamente en el control ex post basado en el SIMCE (la amenaza de cerrar escuelas que no logren ciertos puntajes). Hasta aquí no ha habido apertura para implementar en Chile regulaciones que son ampliamente usadas en países de la OECD, como la racionalización de la distribución geográfica de la oferta escolar, la garantía de cierta eficiencia de los establecimientos (por ejemplo, exigiendo un tamaño mínimo para recibir subvención) o la exigencia de demostrar estándares de calidad previo a recibir subvención estatal.

En síntesis: en los últimos años se ha logrado un amplio acuerdo en Chile en que el mercado no logra satisfacer los propósitos de hacer más competitiva, más justa y de calidad la educación escolar, por lo que la institucionalidad educativa y la política educacional juegan un rol insustituible. Consecuentemente, las reformas institucionales como la Ley General de Educación y el Sistema Nacional de Aseguramiento de la Calidad están orientadas hacia la configuración de una institucionalidad más 
adecuada, que reposiciona al Estado como garante y promotor de la calidad y la equidad educativas. Sin embargo, también en este nuevo paradigma hay opciones que tomar. A nuestro juicio, las políticas recientes contienen importantes omisiones y están desbalanceadas hacia el polo "aplicación de incentivos y presión" en desmedro del eje "apoyo a la generación de capacidades", todo lo cual puede comprometer severamente su efectividad. Ciertamente, el Gobierno está a tiempo para introducir modificaciones que permitan equilibrar este desbalance.

\section{Referencias bibliográficas}

Barber, M. y Mourshed, M. (2007). How the world's best performing school systems come out on top. McKinsey \& Company Report.

Bellei, C. (2009). "The Private-Public School Controversy: The Case of Chile”. En Paul Peterson y Rajashri Chakrabarti (editores), School Choice International. Massachusetts: MIT Press.

Bellei, C. (2010). “Evolución de las políticas educacionales en Chile: 1980-2009”. En Bilbao A. y Salinas A. (editores) El libro abierto de la informática educativa. Santiago: Ministerio de Educación.

Bellei, C. y Valenzuela, J. P. (2010). “¿Están las condiciones para que la docencia sea una profesión de alto estatus en Chile?”. En Martinic, S., y Elacqua, G. (editores), Fin de Ciclo: Cambios en la Gobernanza del Sistema Educativo. Santiago: Facultad de Educación, Pontificia Universidad Católica de Chile y Oficina Regional para América Latina y el Caribe UNESCO.

Bellei, C.; Contreras D. y Valenzuela, J. P. (editores) (2008). La agenda pendiente en educación. Profesores, administradores y recursos. Santiago: Editorial Ocholibros.

Bellei, C.; Contreras D. y Valenzuela, J. P. (editores) (2010). Ecos de la revolución pingüina. Santiago: Editorial Pehuén.

Brunner, J. J. y Peña, C. (coord.) (2007). La reforma al sistema escolar: aportes para el debate. Santiago: Universidad Diego Portales y Universidad Adolfo Ibáñez.

Casassus, J. (2010). “Las Reformas Basadas en Estándares: un camino equivocado”. En Bellei, C.; Contreras D. y Valenzuela, J. P. (editores). Ecos de la revolución pingüina. Santiago: Editorial Pehuén.

Consejo Asesor Presidencial para la Calidad de la Educación (2006). Informe Final. Santiago: Consejo Asesor Presidencial para la Calidad de la Educación. 
Consejo Asesor Presidencial para la Educación Superior (2008). Los desafios de la educación superior. Informe del Consejo Asesor Presidencial para la Educación Superior.

Cox, C. (2003). "Las políticas educacionales de Chile en las últimas dos décadas del siglo XX”. En Cox, Cristián (editor), Políticas educacionales en el cambio de siglo. Santiago: Editorial Universitaria.

Cox, C.; Meckes, L. y Bascopé, M. (2010). "La institucionalidad formadora de profesores en Chile en la década del 2000: velocidad del mercado y parsimonia de las políticas". Revista Pensamiento Educativo, Vol. 46 y 47, pp. 205-245.

Elacqua, G.; Martínez, M. y Aninat, C. (2010). “Cómo fortalecer la educación municipal? Capacidad y responsabilidad política”. En Martinic, S. y Elacqua, G. (editores). Fin de Ciclo: Cambios en la Gobernanza del Sistema Educativo. Santiago: Facultad de Educación, Pontificia Universidad Católica de Chile y Oficina Regional para América Latina y el Caribe UNESCO.

Espínola,V. y Claro, J. P. (2010). "El Sistema Nacional de Aseguramiento de la Calidad: Una Reforma Basada en Estándares”. En Bellei, C.; Contreras D. y Valenzuela, J. P. (editores). Ecos de la revolución pingüina. Santiago: Editorial Pehuén.

García-Huidobro, J. y Bellei, C. (2010). “Debate sobre la calidad de la educación: ¿tropiezo u oportunidad?”. Revista Mensaje,Vol. 59, N 589, pp. 202-207.

García-Huidobro, J. (2010). "Para hacer pública la educación pública”. En Martinic, S., y Elacqua, G. (editores). Fin de Ciclo: Cambios en la Gobernanza del Sistema Educativo. Santiago: Facultad de Educación, Pontificia Universidad Católica de Chile y Oficina Regional para América Latina y el Caribe UNESCO.

Hanushek, E. y Raymond, M. (2002). "Sorting out accountability systems”. En Evers W. y Walberg H. (editores). School Accountability. California: Hoover Institution Press.

Hunt, B. (2009). Efectividad del desempeño docente. Una reseña de la literatura internacional y su relevancia para mejorar la educación en América Latina. Washington: PREAL.

Koretz, D. (2008). Measuring up. What Educational Testing Really Tells Us. Estados Unidos: Harvard University Press.

Larroulet, C. y Montt, P. (2010). "Políticas educativas de largo plazo y acuerdo amplio en educación: el caso chileno". En Martinic, S., y Elacqua, G. (editores), Fin de Ciclo: Cambios en la Gobernanza del Sistema Educativo. Santiago: Facultad de Educación, Pontificia Universidad Católica de Chile y Oficina Regional para América Latina y el Caribe UNESCO.

Manzi, J. (2010). "Programa INICIA: fundamentos y primeros avances". En Bellei, C.; Contreras D. y Valenzuela, J. P. (editores). Ecos de la revolución pingüina. Santiago: Editorial Pehuén. 
Marcel, M. et al. (2009). "Propuestas para una estrategia de revalidación de la educación pública de gestión local”. En Marcel, M. y Raczynski, D. (editores), La asignatura pendiente. Santiago: Uqbar / CIEPLAN.

Ministerio de Educación (2010). Estadísticas de la Educación en Chile 2009. Santiago: Departamento de Estudios y Desarrollo.

OECD (2009). Los docentes son importantes: atraer, formar y conservar a los docentes eficientes. OECD.

Ravela, P. et al. (2008). Las Evaluaciones Educativas que América Latina Necesita. Washington: PREAL.

Umansky, I. (2005). "A Literature Review of Teacher Quality and Incentives. Theory and Evidence". En Vegas, Emiliana (editor), Incentives to improve teaching. Washington: The World Bank.

UNESCO (2000). Aptitudes básicas para el mundo de mañana. Otros Resultados del Proyecto Pisa. Montreal: OCDE/UNESCO-VIS.

Vanni, X. y Bravo, J. (2010). "En búsqueda de una educación de calidad para todos: el Sistema Nacional de Aseguramiento de la Calidad”. En Martinic, S., y Elacqua, G. (editores), Fin de Ciclo: Cambios en la Gobernanza del Sistema Educativo. Santiago: Facultad de Educación, Pontificia Universidad Católica de Chile y Oficina Regional para América Latina y el Caribe UNESCO. 


\section{Anexo}

\section{Compromisos Discurso 21 de mayo 2010 en el Área Educación}

\begin{tabular}{|l|}
\hline \multicolumn{1}{|c|}{ COMPROMISOS SISTEMA ESCOLAR } \\
\hline “... sacar adelante el proyecto que promueve la calidad de la educación y crea una nueva \\
institucionalidad educacional... Este proyecto crea una agencia que evaluará a los colegios \\
y una superintendencia que fiscalizará el correcto uso de los recursos..." \\
\hline “...tendremos funcionando en marzo del 2011 los primeros quince Liceos Bicentenarios, \\
de un total de cincuenta liceos de excelencia mixtos en las principales ciudades de Chile, \\
para jóvenes esforzados y talentosos." \\
"Tercero, estamos comprometidos a duplicar la subvención educacional en un plazo de \\
ocho años." \\
"Pese a que este es un año difícil, cumpliremos este compromiso ahora mismo incre- \\
mentando la subvención para los alumnos más vulnerables." \\
“...en los barrios más afectados por la droga y la delincuencia, mantendremos abiertos los \\
colegios municipales hasta las ocho de la noche, para que los niños y jóvenes que quieran \\
no tengan que quedarse en la calle o solos en sus casas." \\
“...Para ello, les enviaremos por correo, junto con una carta personal del Presidente de la \\
República, los resultados de la próxima prueba Simce, por colegio a todos los apoderados, \\
y no sólo a los del curso que dio la prueba."
\end{tabular}

"Además, estableceremos premios e incentivos para aquellos alumnos, profesores y escuelas que logren mejorar notoriamente sus rendimientos."

“Este año, cuando se tome la prueba Simce, se agregará una prueba de inglés."

“...y a futuro, una de educación fisica y de conocimientos de la tecnología de la información."

"Crearemos programas especiales de apoyo para aquellas escuelas con resultados insuficientes. Para ello, lanzaremos dos programas: "Comprométete con una Escuela" y "Comprométete con un Niño."

"Para lograrlo, un panel de expertos ya está trabajando en un proyecto de nueva carrera Docente, que enviaremos al Congreso."

"Propondremos incentivos para que alumnos de muy buenos puntajes en la PSU quieran ser profesores."

"Trabajaremos en conjunto con las universidades para mejorar la formación y capacitación de los profesores."

"Queremos que la prueba Inicia, que se da al egresar de la carrera docente, deje de ser voluntaria y sus resultados sean conocidos y tengan consecuencias."

"Fortaleceremos el liderazgo de los directores y las remuneraciones de los buenos profesores." 


\section{COMPROMISOS SISTEMA ESCOLAR}

"También le hemos pedido a este panel que busque nuevos y mejores modelos para la educación municipal."

“...quiero manifestar mi preocupación por el clima y la convivencia escolar. El respeto y la tolerancia deben no sólo enseñarse, sino también practicarse. Adicionalmente, aumentaremos las exigencias a nuestros alumnos, estableciendo un mejor equilibrio entre sus derechos y obligaciones."

"Daremos a conocer en los próximos días un Servicio País para la educación, que permitirá a jóvenes profesionales, hacer clases en escuelas vulnerables."

\begin{tabular}{|l|}
\hline COMPROMISOS EDUCACIÓN PRE-ESCOLAR \\
\hline “...vamos a llevar la educación preescolar hasta el último rincón del país...” \\
\hline “...y ampliaremos los horarios de atención de salas cunas y jardines infantiles." \\
\hline \multicolumn{1}{c|}{ COMPROMISOS EDUCACIÓN SUPERIOR } \\
\hline $\begin{array}{l}\text { "Seguiremos mejorando nuestro sistema de educación superior y haremos que los aportes } \\
\text { del Estado a las universidades tengan como contrapartida mejorías en la calidad de sus } \\
\text { facultades pedagógicas y su aporte a la ciencia y tecnología." }\end{array}$ \\
\hline $\begin{array}{l}\text { "Implementaremos un ambicioso programa de becas de magíster y doctorados, asegurando } \\
\text { la excelencia de los postulantes e incrementando las exigencias para su vuelta a Chile." }\end{array}$ \\
\hline
\end{tabular}

\section{Compromisos Programa de Gobierno 2010 - 2014}

\begin{tabular}{|l|}
\hline \multicolumn{1}{|c|}{ COMPROMISOS EDUCACIÓN PRE-ESCOLAR } \\
\hline $\begin{array}{l}\text { "En relación a la educación pre-escolar, nuestra meta es llegar a todos los rincones del país, } \\
\text { aumentando la cobertura especialmente en los sectores de menores ingresos, asegurándo- } \\
\text { nos así que se logren eficazmente los desarrollos cognitivos y no cognitivos requeridos } \\
\text { para una educación de calidad". }\end{array}$ \\
\hline \multicolumn{1}{c|}{ COMPROMISOS SISTEMA ESCOLAR } \\
\hline $\begin{array}{l}\text { "Duplicaremos gradualmente la subvención escolar por niño atendido y crear una insti- } \\
\text { tucionalidad para que expertos definan periódicamente los niveles y valores que deben } \\
\text { alcanzar los diferentes tipos de subvenciones". }\end{array}$ \\
"Asimismo, se promoverá una subvención a la calidad que premie a los establecimientos y \\
equipos docentes que se destaquen a nivel país". \\
"Elevaremos las exigencias a los establecimientos, a sus directivos, docentes y alumnos, lo \\
que será coordinado por la nueva Agencia de Calidad de la Educación. Se incorporarán \\
nuevas plataformas tecnológicas para apoyar a directores, profesores y alumnos, posibili- \\
tando una verdadera revolución digital al interior de la sala de clases".
\end{tabular}


"Crearemos una unidad especializada de apoyo a los establecimientos que lo soliciten, la cual además certificará a las agencias independientes de apoyo que deseen participar en el proceso".

"Se premiará a los cursos que muestren mejoras significativas en el aprendizaje con viajes culturales, materiales educativos de calidad, etc. Asimismo, se mejorará la calidad de los textos escolares colocándolos en línea con los mejores textos a nivel mundial".

"Se apoyarán las iniciativas destinadas a mejorar el clima al interior de las escuelas, liceos y colegios del país. Concordante con ello existirá tolerancia cero con la violencia, las drogas y la falta de respeto a las jerarquías en nuestros establecimientos escolares".

"Favoreceremos la movilidad social y la verdadera igualdad de oportunidades en la educación. Para lograr lo anterior se contemplan políticas y programas como, la creación de una red de a lo menos 50 liceos de excelencia como el Instituto Nacional; la continuación y profundización de la subvención preferencial para los estudiantes de familias más desvalidas; así como el fomento a las familias que aportan financiamiento compartido. Además, se otorgarán incentivos para que los buenos profesores desarrollen su carrera educando alumnos en situación de vulnerabilidad".

"Crearemos una red nacional de docentes expertos para apoyar a los niños y niñas que se estén quedando atrás en los procesos de aprendizaje”.

"En materia de educación media técnico-profesional, se perfeccionará el proceso de enseñanza, en el sentido de alinearla en torno a las competencias laborales que el sector productivo y de servicios requieren, y asimismo, se realizarán las coordinaciones y compatibilizaciones necesarias para una integración con la educación superior de carácter técnico”.

"Mejoraremos la calidad de los directores de los establecimientos escolares. Para ello se crearán comités de búsqueda para seleccionar a quienes, con habilidades de liderazgo, muestren las mejores condiciones para ser directores de establecimientos educacionales".

"Se promoverán programas de formación en las mejores universidades, integrando las tareas de las escuelas de pedagogías con las escuelas de administración. Se pondrá término a los cargos vitalicios de directores, evolucionándose hacia un sistema de selección por méritos. Los concursos para seleccionar directores se perfeccionarán, elevando los requisitos para los participantes y profesionalizando la integración del comité de búsqueda”.

"El panel de expertos, que evaluará periódicamente el valor apropiado de la subvención escolar, realizará también una estimación del adecuado nivel de remuneraciones y de incentivos para los nuevos directores de los establecimientos escolares".

"Atraeremos a los jóvenes talentosos a la profesión docente y retener a los profesores más efectivos. Para hacerlo se crearán becas atractivas para financiar estas carreras a aquellos alumnos de altos puntajes que se matriculen en pedagogía. Estos beneficios podrán utilizarse sólo en carreras con un nivel elevado de acreditación. Los buenos alumnos tendrán prioridad para acceder a la profesión docente. Se aumentarán los estándares de acreditación de las carreras de pedagogía". 
"Se establecerá un examen no obligatorio de habilitación. Aquellos egresados que no hayan rendido examen de habilitación o que no tengan una buena calificación, y que ingresen a la profesión docente, quedarán facultados para ejercer la carrera sólo en forma condicional y deberán demostrar efectividad en la sala de clase. A medida que vayan superando ciertas metas podrán aspirar a las remuneraciones de quienes obtuvieron buenas calificaciones en los exámenes. Cada tres años deberán verificarse las capacidades docentes en la sala de clases. Este proceso será realizado en forma descentralizada, y sus criterios generales de evaluación serán dirigidos por la Agencia de Calidad de Educación. Los nuevos directores de los establecimientos podrán solicitar el reentrenamiento de los profesores".

"Crearemos una nueva carrera docente que apoye a los profesores en su tarea de mejorar los aprendizajes y la formación de sus alumnos. El actual Estatuto Docente no lo permite, debido a que contiene excesivas rigideces. Se diseñarán mecanismos para mejorar las remuneraciones vinculadas a los aprendizajes de los estudiantes, y para relacionar el nivel de remuneraciones con la necesidad de atraer a la profesión docente a personas de altas habilidades. Además, se establecerán programas especiales de perfeccionamiento para las personas que no logren las metas establecidas por cada unidad escolar. Otra modificación consistirá en premiar con bonos adicionales a los docentes que desarrollen labores en escuelas, liceos o colegios con un número alto de niños o jóvenes vulnerables. La actual normativa se modificará gradualmente. Aquellos profesores que no tengan la calificación adecuada contarán con los mecanismos para acceder a programas de reentrenamiento. Asimismo, gradualmente se irán descentralizando las evaluaciones periódicas, siguiendo las normas definidas por la Agencia de Calidad de la Educación”.

"Avanzaremos hacia la meta de hacer de Chile un país bilingüe... Para ello crearemos un programa bicentenario que contiene tres elementos: financiar buenos programas de formación de docentes en inglés, creando para ello un fondo concursable; crear un programa de capacitación para profesores de inglés con capacidades insuficientes; y traer al país, a través de concursos internacionales, a profesores extranjeros con dominio del idioma inglés".

"Estableceremos un sistema educativo integrado con el mundo digital... proponemos dotar gradualmente todas las salas de clases del país con un computador y un data show, una pizarra interactiva y conexión a internet de banda ancha, de modo de aprovechar la tecnología para el desarrollo de las clases. Además, se promoverán plataformas digitales que permitan mejorar la gestión administrativa y el control de asistencia a clases a través de indicadores biométricos. Esta plataforma contribuirá también a apoyar a los profesores y los estudiantes".

"Elevaremos los estándares de aprendizaje y aumentar la información a las familias respecto de la calidad de los establecimientos educacionales... se definirán estándares de aprendizaje precisos, que tendrán que ser satisfechos por los establecimientos en períodos de tiempo razonables. Lo anterior será complementado con las visitas periódicas de los expertos de la Agencia de Calidad de Educación, los que elaborarán un informe que será de conocimiento público y que se enviará periódicamente a los familiares, y que tomará en cuenta la auto-evaluación previa del establecimiento".

"Se establecerá un programa de apoyo para mejorar los desempeños en aquellos establecimientos que lo soliciten. Éste será obligatorio para los que no alcancen los estándares prioritarios. En el caso de aquellos establecimientos que no satisfagan los estándares mínimos en plazos razonables, dejarán de ser sujetos de la subvención". 
“Anualmente los sostenedores deberán rendir cuenta a los padres y apoderados acerca de los desempeños de sus hijos. Asimismo, esta información deberá contener los objetivos de aprendizaje para los próximos cuatro años y los instrumentos que se pretende utilizar para alcanzar esas metas".

"Para mejorar la información disponible de los padres en el proceso de elección de un establecimiento educacional para que concurran sus hijos, se exigirá la entrega de cartillas de información donde los apoderados puedan apreciar, en forma simple y clara, los resultados de sus hijos en comparación a otros establecimientos de la localidad en que viven, para facilitar así decisiones más informadas en beneficio de los niños".

"Mejoraremos la educación pública municipal. Proponemos la creación de Corporaciones de Educación de Calidad (CEC) a nivel comunal, las que tendrán como finalidad única el mejoramiento de la calidad de la educación en la respectiva comuna. El directorio de la Corporación será presidido por el Alcalde, siendo éste la autoridad máxima y por lo tanto el responsable de los respectivos resultados. Esta Corporación otorgará el sustento administrativo y coordinará la gestión técnica pedagógica. El director ejecutivo de la corporación será seleccionado por méritos con criterios similares a los que utiliza el Sistema de Alta Dirección Pública. Estas corporaciones podrán asociarse con las de otras comunas para aprovechar economías de escala, lo cual es particularmente importante en el caso de las comunas pequeñas. En el caso de comunas grandes, podrá haber más de una corporación”.

\section{COMPROMISOS EDUCACIÓN SUPERIOR}

"Para lograr igualdad de oportunidades en el acceso a la educación superior se perfeccionará el sistema de financiamiento estudiantil ampliando su acceso, eliminando las discriminaciones injustificadas y focalizando las becas en los sectores de menores ingresos y para aquellas carreras de mayor retorno social, incluyendo especialmente las pedagogías".

"Mejoraremos el sistema de becas aumentando su equidad horizontal y vertical, terminando con la discriminación en el financiamiento según la naturaleza jurídica de la institución de educación superior elegida por el alumno. Se aumentarán las becas para las carreras de alta valorización social y para los sectores de menores ingresos. Se eliminarán gradualmente las diferencias en los sistemas de créditos y se perfeccionará un seguro para que los jóvenes puedan continuar sus estudios cuando, a raíz del desempleo, tengan problemas para financiarlos".

"Se perfeccionará la institucionalidad del sector. Existirá una entidad que agrupará a los institutos profesionales y centros de formación técnica, y otra que agrupará a las universidades".

"Se promoverá la renovación curricular incentivando un esquema de formación general con especialización posterior, con carreras más breves y con una mayor articulación al interior del sistema para promover la movilidad estudiantil.".

"Se fortalecerá el sistema de acreditación adoptando medidas especiales para las carreras de pedagogía, y con un mayor desarrollo de las agencias acreditadoras".

"Se extenderá el programa MECESUP más allá del 2011". 
"se modificará gradualmente el aporte fiscal directo, de manera de condicionarlo al cumplimiento de ciertos criterios de excelencia en su desempeño... abrir esta fuente de recursos a todas las instituciones universitarias que satisfagan tales requisitos".

"En el caso de las universidades estatales se realizará una reforma que les permita eliminar trabas burocráticas y flexibilizar su gestión académica, financiera y administrativa, de forma de hacerlas plenamente competitivas. Se les permitirá acceder a mayores recursos para inversión utilizando mecanismos como las concesiones de obras, y se las dotará de nuevos gobiernos corporativos para aumentar su vinculación con entidades regionales, sectores productivos y otros, logrando así un mayor acceso a financiamiento a través de proyectos y donaciones".

"Se aumentará la investigación científica y tecnológica en el mundo de la educación superior. Para ello, se intensificará la formación de capital humano incrementando el número de becas para programas de doctorado y post doctorado; se aumentarán los recursos para INNOVA y FONDEF; se fortalecerá el rol y aumentarán al doble los proyectos financiados a través de FONDECYT, y se continuará incentivando a los centros de excelencia”.

"se perfeccionará la institucionalidad encargada del desarrollo científico y tecnológico, eliminando fallas de coordinación y unificando criterios para la formación de fondos concursables. Se le dará más autonomía y visión de largo plazo a CONICYT, se creará una agencia de innovación tecnológica y se rediseñará el Consejo de Innovación, para dar una mayor participación a las universidades y para que pueda contar con un consejo asesor de científicos a nivel internacional".

"perfeccionar el crédito fiscal de 35\% para inversión privada en investigación y desarrollo, eliminando las restricciones excesivas que hoy lo afectan y que dificultan la investigación realizada al interior de la empresa o financiada por ésta. 